[0212-7199 (2005) 22: 11; pp 520-524] ANALES DE MEDICINA INTERNA Copyright $\odot 2005$ ARAN EDICIONES, S.L.

AN. MED. INTERNA (Madrid) Vol. 22, N. ${ }^{\circ} 11$, pp. 520-524, 2005

\section{Grado de cumplimiento de los objetivos de control metabólico y de los factores de riesgo cardiovascular en pacientes diabéticos tipo 2 en un área de Atención Primaria}

\author{
E. GONZÁLEZ SARMIENTO, B. VIELBA DUEÑAS ${ }^{1}$, I. FERNÁNDEZ GALANTE, \\ S. MANSO GARCÍA ${ }^{1}$, Y. SAN PEDRO MARTÍN ${ }^{1}$, D. VIELBA DUEÑAS, \\ R. FERNÁNDEZ GONZÁLEZ1
}

Servicio de Medicina Interna. Hospital Universitario. ${ }^{1}$ Centro de Salud de Rondilla 2. Área Este de Atención Primaria. Valladolid

\section{RESUMEN}

La diabetes mellitus tipo 2 (DM2) representa un importante problema de salud pública, debido a su elevada prevalencia y alta morbimortalidad, que se incrementa, aún más, por su asociación a determinados factores de riesgo cardiovascular (FRV). Por ello es importante una acción conjunta sobre todas estas alteraciones, ya que su control previene las manifestaciones vasculares.

Objetivo: Se basa en la detección y determinación del grado de control de los factores de riesgo asociados a pacientes con DM2, según los datos dados por las diferentes organizaciones internacionales, estudiados en un Centro de Salud de Valladolid.

Método: Se trata de un estudio transversal descriptivo para evaluar el control de distintos FRV en pacientes diagnosticados y tratados de DM2 de acuerdo a los diferentes criterios establecidos. La muestra está formada por 74 pacientes adultos (41 varones y 33 mujeres), incluidos en el Programa de Diabetes Mellitus de un "cupo" perteneciente al Centro de Salud de Rondilla 2, del Área Este de Atención Primaria de Valladolid, para la consulta programada de control de riesgo cardiovascular.

Resultados y conclusiones: Se detecta un pobre control de la mayoría de los FRV, por lo que deberán indicarse medidas de control más estrictas, con el fin de prevenir las complicaciones vasculares relacionadas con ellos.

PALABRAS CLAVES: DM tipo 2. FRV. Objetivos control.
DEGREE OF FULFILLMENT OF THE OBJECTIVES OF CONTROL METABOLICO AND THE FACTORS OF DIABETIC CARDIOVASCULAR RISK IN PATIENTS TYPE 2 IN AN AREA OF PRIMARY ATTENTION

\section{ABSTRACT}

Diabetes mellitus type is one of de most important health problem in the world, due to its high prevalence and morbidity and its relation with several cardiovascular risk factors. That's why a global action, aimed to prevent these vascular syndromes, is needed.

Objective: the goal of this study is to detect and determine how cardiovascular risk factor are controlled in diabetic type 2 patients, according to the date supplied by several international organization that have been studied at a Health Centre of Valladolid.

Methods: It is a descriptive cross-sectional study to evaluate the control of several cardiovascular risk factors in diabetic patients according to the different stablished criteria. The sample is formed by 74 adult patients (41 men and 33 women), included in the Diabetes Mellitus Programm at Health Centre of Rondilla 2, at East Area of Primary Care of Valladolid, in the programmed consultation of cardiovascular risk factors control.

Results and conclusion: I has been observed that the majority of these cardiovascular risk factors are not well controlled. In this way, stringent mesures of control should be considered in order to prevent the cardiovascular complications related to them.

KEY WORDS: DM type 2. Cardiovascular risk factors.

González Sarmiento E, Vielba Dueñas B, Fernández Galante I, Manso García S, San Pedro Martín Y, Vielba Dueñas D, Fernández González $R$. Grado de cumplimiento de los objetivos de control metabólico y de los factores de riesgo cardiovascular en pacientes diabéticos tipo 2 en un área de Atención Primaria. An Med Interna (Madrid) 2005; 22: 520-524.

\section{INTRODUCIÓN}

La diabete mellitus tipo 2 (DM2) es un proceso de elevada prevalencia y alta morbimortalidad. Afecta a más del $3 \%$ de las personas adultas y al 6\% de la población entre 30-60 años (1). Las previsiones para el año 2025 son de 300 millones de diabéticos en todo el mundo, hablándose ya de la epidemia del siglo XXI (2).

Supone la sexta causa de muerte en España, ocurriendo más del $90 \%$ por encima de los 65 años (1), principalmente por enfermedad cardiovascular. Esta representa el 70-80\% de todas las causas de muerte en la DM $(3,4)$ e incrementa en 2-4 veces la incidencia de infarto de miocardio. Se debe a la acción individualizada o conjunta de determinados FRV asociados a este proceso, como hipertensión arterial, dislipemia, obesidad etc. Esto hace que la DM2 sea un importante problema asistencial, de salud y económico.

Por todo ello se hace necesario profundizar en su buen control metabólico y en el de los FRV que más influyen en su evolución, para intentar prevenir o frenar el desarrollo de estas

Trabajo aceptado: 16 de septiembre de 2005

Correspondencia: E. González Sarmiento. Hospital Clínico Universitario de Valladolid. Planta 10ª Este. Ramón y Cajal, 3. 47005 Valladolid. e-mail: enrgonz@med.uva.es 
complicaciones $(5,6)$. A pesar de los esfuerzos realizados estamos aún lejos de conseguir estos objetivos, por lo que hay que insistir en los diferentes niveles sanitarios para lograr nuevas estrategias terapéuticas para alcanzarlos.

El objetivo de este estudio ha sido evaluar el grado de control metabólico y de los múltiples factores de riesgo cardiovascular asociados, tanto clásicos como emergentes, en una muestra de pacientes con DM2 pertenecientes a un Centro de Salud de Atención Primaria, comparándolos con los objetivos de control óptimo según las recomendaciones dadas por los diferentes consensos internacionales.

\section{MATERIAL Y METODOS}

Se trata de un estudio transversal descriptivo para evaluar el control de distintos FRV en pacientes con DM2. La muestra está formada por 74 pacientes adultos (41 varones y 33 mujeres), incluidos en el Programa de Diabetes Mellitus de un "cupo" perteneciente al Centro de Salud de Rondilla 2, del Área Este de Atención Primaria de Valladolid, para la consulta programada de control de riesgo cardiovascular.

Los criterios de inclusión han sido: pacientes con DM2 independientemente de la raza, sexo, grado de control metabólico y tipo de tratamiento. Se han excluido los pacientes diagnosticados de otro tipo de DM.

A todos los pacientes, aparte de las determinaciones sanguíneas de rutina que incluían un hemograma completo, glucemia basal, lipidograma, electrolitos, urea, creatinina y microalbuminuria, se les determinó HbA1c y glucemia posprandial (GPP) en sangre capilar, mediante reflectómetro, tomada por el propio paciente en su domicilio, valorándose el último valor recogido por el paciente a las 2 horas del desayuno.

Como índices antropométricos se valoraron el peso y la talla, índice de masa corporal (IMC), la circunferencia de la cintura (CC), de la cadera y el índice cintura cadera (ICC). Así mismo se valoró la presión arterial sistólica y diastólica (PAS y PAD) que se obtuvieron mediante la utilización de una técnica braquial estándar, con el paciente sentado y relajado, tras 10 minutos de reposo. Se utilizó la media de dos determinaciones.

De la historia clínica fueron recogidos los antecedentes familiares de riesgo cardiovascular así como el consumo de tabaco, alcohol y las medicaciones que recibían en el momento de la recogida de los datos.

Objetivos de control: el grado de control se realizó siguiendo los parámetros dados por las diferentes Guias Internacionales: Asociación Americana de Diabetes (ADA) (7), European Diabetes Poly Group (8), National Colesterol Education Program, Adult Tratmente Panel III (ATPIII) (9), UKPDS (5), Joint National Comité on Prevention, Detection, Evaluation and Treatment of High Blood Pressure, The 7th Report of de Joint National Committee on Prevention Detection, Evaluation and Treatmen og High Blood Pressure (VII $J N C$ ) (10) y el National Institute of Health (11). Según ellos se han aceptado los siguientes objetivos de control: GB $<120$ $\mathrm{mg} / \mathrm{dl}, \mathrm{GPP}<160 \mathrm{mg} / \mathrm{dl}, \mathrm{HbA} 1 \mathrm{c}<7 \%$, colesterol total $<200$ $\mathrm{mg} / \mathrm{dl}$, LDL-colesterol $<100 \mathrm{mg} / \mathrm{dl}$, HDL-colesterol $>45$ $\mathrm{mg} / \mathrm{dl}$ en varones y $>55 \mathrm{mg} / \mathrm{dl}$ en mujeres, $\mathrm{TG}<200 \mathrm{mg} / \mathrm{dl}$, PAS < $130 \mathrm{mmHg}$, PAD < $85 \mathrm{mmHg}$, IMC $<25 \mathrm{~m} / \mathrm{kg} 2, \mathrm{CC}<$ $102 \mathrm{~cm}$ en varones $\mathrm{y}<88 \mathrm{~cm}$ en mujeres, ICC $<1$ en varones y $<0,88$ en mujeres, ausencia de hábito tabáquico.
Las medicaciones valoradas en este estudio fueron: antidiabéticos orales (ADO), insulina, estatinas, inhibidores de la enzima conversora de la angiotensina (IECAS), calcioantagonistas, inhibidores de los receptores de la angiotensina (ARAII), betabloqueantes y antiagregantes plaquetarios.

El análisis estadístico se ha realizado con el programa SPSS versión 115 para windows. Se ha utilizado el test chicuadrado de Pearson para comparar las variables categóricas. En variables continuas las diferencias entre medias se han comparado con la t de Student. Se ha considerado como significativo una $\mathrm{p}<0.05$.

\section{RESULTADOS}

Las características basales de los pacientes estudiados se muestran en la tabla I. Las medias y desviación estándar se exponen de forma global y en relación al sexo. Se aprecian diferencias significativas en cuanto al sexo en el peso, talla ( $p$ $<0,01)$, circunferencia de la cintura $(\mathrm{p}<0,05)$, ICC $(\mathrm{p}<$ $0,01)$, glucemia basal y creatinina $(\mathrm{p}<0,05)$.

\section{TABLA I}

CARACTERISTICAS BASALES DE LOS PACIENTES CON DM TIPO 2 ESTUDIADOS

\begin{tabular}{lcccc}
\hline Características & Total & Varones & Mujeres & $p^{*}$ \\
\hline N $^{0}$ pacientes & 74 & $41(55,4 \%)$ & $33(44,6 \%)$ & NS \\
Edad (años) & $67,86 \pm 13,10$ & $65,51 \pm 13,46$ & $70,87 \pm 12,38$ & NS \\
Peso (kg) & $73,71 \pm 12,57$ & $77,10 \pm 11,72$ & $69,48 \pm 12,67$ & $<0,01$ \\
Talla (m) & $1,60 \pm 0,09$ & $1,65 \pm 0,08$ & $1,55 \pm 0,07$ & $<0,01$ \\
IMC (m/kg $)$ & $28,67 \pm 4,82$ & $28,36 \pm 4,25$ & $28,98 \pm 5,56$ & NS \\
C, cintura (cm) & $98,07 \pm 11,76$ & $97,12 \pm 9,45$ & $99,30 \pm 14,70<0,05$ \\
C, cadera (cm) & $102,48 \pm 8,35$ & $102,15 \pm 7,94$ & $102,9 \pm 9,27$ & NS \\
ICC & $0,95 \pm 0,06$ & $0,95 \pm 0,06$ & $0,96 \pm 0,07$ & $<0,01$ \\
PAS (mmHg) & $131,3 \pm 18,37$ & $128,74 \pm 18,01$ & $134,43 \pm 18,93$ & NS \\
PAD (mmHg) & $75,3 \pm 10,75$ & $74,61 \pm 10,03$ & $76,37 \pm 11,83$ & NS \\
CB (mg/dl) & $136,94 \pm 40,86$ & $148,07 \pm 48,38$ & $120,91 \pm 1,35<0,05$ \\
CPP ((mg/dl) & $144,10 \pm 39,87$ & $135,89 \pm 20,57$ & $146,59 \pm 41,08$ & NS \\
HbA1c (\%) & $6,59 \pm 1,52$ & $6,72 \pm 1,65$ & $6,39 \pm 1,35$ & NS \\
HDL-C (mg/dl) & $56,22 \pm 18,44$ & $55,02 \pm 19,82$ & $57,78 \pm 17,05$ & NS \\
LDL-C (mg/dl) & $130,54 \pm 104,91$ & $118 \pm 31$ & $147,02 \pm 154,78$ & NS \\
CT (mg/dl) & $191,88 \pm 41,11$ & $185,5 \pm 44,58$ & $199,06 \pm 36,43$ & NS \\
TC (mg/dl) & $107,79 \pm 52,53$ & $180,8 \pm 58,58$ & $107,69 \pm 45,25$ & NS \\
Creatinina (mg/dl) & $0,89 \pm 0,25$ & $0,98 \pm 0,22$ & $0,77 \pm 0,23$ & $<0,05$ \\
$\mu$ albuminuria (mg/día) & $153,55 \pm 357,22$ & $145,88 \pm 241,59$ & $163,41 \pm 477,27$ & NS \\
\hline
\end{tabular}

* = valor de $\mathrm{p}$ entre las mujeres y los varones; NS = no significativo; IMC = índice de masa corporal; ICC = índice cintura-cadera; PAS/PAD = Presión arterial sistólica y diastólica; $\mathrm{GB}=$ glucemia basal, $\mathrm{GPP}=$ glucemia posprandial, $\mathrm{CT}=$ colesterol total; $\mathrm{TG}=$ triglicéridos.

Los diferentes factores de riesgo asociados y los antecedentes familiares en ambos sexos se exponen en la tabla II. Hay diferencias significativas en los porcentajes de HTA, que es el más prevalente después de la obesidad, con un $60 \%$ de los pacientes, antecedentes familiares de HTA y de ACVA que son mayores en las mujeres $(\mathrm{p}<0,05)$ y de consumo de alcohol y de tabaco que es mayor en los hombres $(\mathrm{p}<0,05)$.

En cuanto al tipo de tratamiento utilizado no existen diferencias significativas entre ambos sexos. Los porcentajes se exponen en la tabla III. 
TABLA II

\section{PORCENTAJES DE LOS DISTINTOS FACTORES DE RIESGO ASOCIADOS A LA DM TIPO 2 EN LA POBLACIÓN ESTUDIADA}

\begin{tabular}{lcccc}
\hline Caracteristicas & Total & Varones & Mujeres & $p^{*}$ \\
\hline Obesidad & $57(76 \%)$ & $31(75,6 \%)$ & $26(78,1 \%)$ & NS \\
HTA & $45(60 \%)$ & $19(46,3 \%)$ & $25(75,8 \%)$ & $<0,05$ \\
Dislipemia & $20(40 \%)$ & $13(46,4 \%)$ & $7(33,3 \%)$ & NS \\
ualbuminuria & $17(53 \%)$ & $11(61 \%)$ & $6(42,9 \%)$ & NS \\
Tabaco & $17(23 \%)$ & $14(35 \%)$ & $3(9,1 \%)$ & $<0,01$ \\
Alcohol & $8(10,8 \%)$ & $8(20 \%)$ & $0(0 \%)$ & $<0.05$ \\
Cl & $5(6,8 \%)$ & $3(7.3 \%)$ & $2(6,3 \%)$ & NS \\
ACVA & $2(2,7 \%)$ & $2(4,9 \%)$ & $0(0 \%)$ & NS \\
AF. HTA & $11(21,6 \%)$ & $3(10,7 \%)$ & $8(36,4 \%)$ & $<0,05$ \\
AF. DM tipo 2 & $18(33,5 \%)$ & $9(32,1 \%)$ & $8(36,4 \%)$ & NS \\
AF. dislipemia & $5(9,8 \%)$ & $2(7,1 \%)$ & $3(13,6 \%)$ & NS \\
AF. Cl & $6(11,8 \%)$ & $4(14,3 \%)$ & $2(9,1 \%)$ & NS \\
AF. ACVA & $6(11,8 \%)$ & $1(3,6 \%)$ & $5(22,7 \%)$ & $<0,05$ \\
\hline
\end{tabular}

$\mathrm{Cl}=$ cardiopatía isquémica; $\mathrm{ACVA}=$ accidente cerebrovascular agudo; $\mathrm{AF}=$ antecedentes familiares.

\section{TABLA III}

PORCENTAJES DE LOS DISTINTOS TRATAMIENTOS FARMACOLÓGICOS PRESCRITOS EN LA MUESTRA ESTUDIADA

\begin{tabular}{lcccc}
\hline Características & Total & Varones & Mujeres & $p^{*}$ \\
\hline ADO & $62 \%$ & $63,2 \%$ & $62,5 \%$ & NS \\
Insulina & $31 \%$ & $29,7 \%$ & $30,3 \%$ & NS \\
ADO/insulina & $8,11 \%$ & $4,05 \%$ & $4,05 \%$ & NS \\
IECA & $27,5 \%$ & $32,1 \%$ & $18,2 \%$ & NS \\
ARAll & $23,5 \%$ & $17,9 \%$ & $31,8 \%$ & NS \\
CA-antagonista & $25,5 \%$ & $21,4 \%$ & $31,8 \%$ & NS \\
Betabloqueante & $7,8 \%$ & $10,7 \%$ & $4,5 \%$ & NS \\
Antiagregantes & $15,7 \%$ & $21,4 \%$ & $9,1 \%$ & NS \\
Estatinas & $18 \%$ & $14,8 \%$ & $22,7 \%$ & NS \\
\hline
\end{tabular}

$\mathrm{ADO}=$ antidiabéticos orales

Con respecto a los objetivos de control los porcentajes de pacientes con criterios de buena compensación se exponen en la tabla IV y en las figuras 1 y 2 , encontrando diferencias significativas en la glucemia basal (25\% varones, $45,5 \%$ mujeres, $\mathrm{p}<0,05$ ), CC (69,2\% varones, $20 \%$ mujeres, $\mathrm{p}<0,05)$ y en el ICC $(84,6 \%$, $0 \%$ respectivamente para hombre y mujeres con $\mathrm{p}<0,01)$.

\section{DISCUSIÓN}

Como consecuencia de los cambios en el estilo de vida, la prevalencia de determinados procesos y, entre ellos la DM2, esta creciendo de manera espectacular. Dada la frecuencia con que la DM2 se asocia a ciertos factores de riesgo cardiovascular, como obesidad, hipertensión arterial y dislipemia, entre otros, hace que sea un importante problema de salud pública. Se sabe, por numerosos estudios (5), que el buen control de la enfermedad y de los factores de riesgo asociados mejoran, de manera evidente, el desarrollo de las alteraciones cardiovasculares. Esto ha llevado a que la mayoría de las sociedades dedicadas a su estudio hallan elaborado una serie de consen-
TABLA IV

PORCENTAJE DE PACIENTES ESTUDIADOS QUE ALCANZAN EL OBJETIVO DE CONTROL ÓPTIMO

\begin{tabular}{|c|c|c|c|c|}
\hline Buen control & Total & Varones & Mujeres & $p^{*}$ \\
\hline $\mathrm{GB}<120 \mathrm{mg} / \mathrm{dl}$ & $33,3 \%$ & $25 \%$ & $45,5 \%$ & $<0,05$ \\
\hline $\mathrm{GPP}<160 \mathrm{mg} / \mathrm{dl}$ & $74,5 \%$ & $82,1 \%$ & $68,2 \%$ & NS \\
\hline $\mathrm{HbA} 1 \mathrm{c}<7 \%$ & $72,2 \%$ & $65 \%$ & $83,9 \%$ & NS \\
\hline HDL-C & & & & NS \\
\hline$>45 \mathrm{mg} / \mathrm{dl}$ en $\mathrm{V}$ & $37,5 \%$ & $37,5 \%$ & & \\
\hline$>55 \mathrm{mg} / \mathrm{dl}$ en $\mathrm{M}$ & $56,3 \%$ & & $56,3 \%$ & \\
\hline LDL-C > $100 \mathrm{mg} / \mathrm{dl}$ & $26,8 \%$ & $25,6 \%$ & $29 \%$ & NS \\
\hline $\mathrm{CT}<200 \mathrm{mg} / \mathrm{dl}$ & $58,9 \%$ & $65 \%$ & $50 \%$ & NS \\
\hline $\mathrm{TC}<200 \mathrm{mg} / \mathrm{dl}$ & $93,2 \%$ & $90 \%$ & $96,9 \%$ & NS \\
\hline$I M C<25 \mathrm{~kg} / \mathrm{m}^{2}$ & $23 \%$ & $24,4 \%$ & $21,9 \%$ & NS \\
\hline $\mathrm{CC}$ & & & & $<0,05$ \\
\hline$<102 \mathrm{~cm}$ en $\mathrm{V}$ & $69,2 \%$ & $69,2 \%$ & & \\
\hline$<88 \mathrm{~cm}$ en $\mathrm{M}$ & $20 \%$ & & $20 \%$ & \\
\hline ICC & & & & $<0,01$ \\
\hline$<1$ en V & $84,6 \%$ & $84,6 \%$ & & \\
\hline$<0,8$ en $M$ & $0 \%$ & & $0 \%$ & \\
\hline $\mathrm{PAS}<135 \mathrm{mmHg}$ & $58 \%$ & $65,8 \%$ & $50 \%$ & NS \\
\hline$P A D<85 \mathrm{mmHg}$ & $82,6 \%$ & $81,6 \%$ & $83,3 \%$ & NS \\
\hline No tabaco & $71,6 \%$ & $55 \%$ & $90,9 \%$ & NS \\
\hline
\end{tabular}

$\mathrm{P}^{*}$ : valor de $\mathrm{p}$ entre las mujeres y los varones. NS: no significativo $\mathrm{V}$ : varones. M: mujeres.

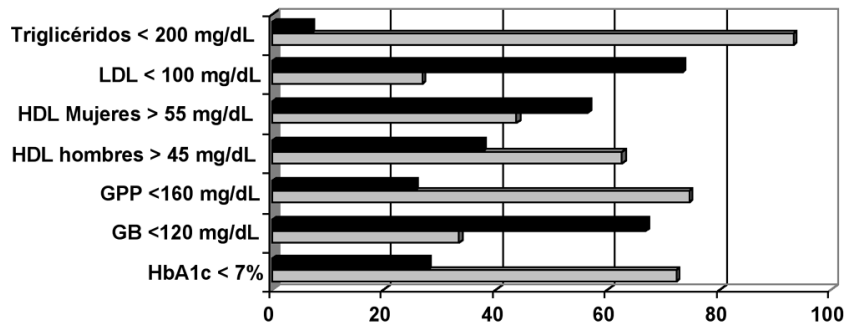

$\square$ Objetivo alcanzado Objetivo no alcanzado

Fig. 1. Porcentaje de pacientes estudiados que alcanzan el objetivo de control óptimo.

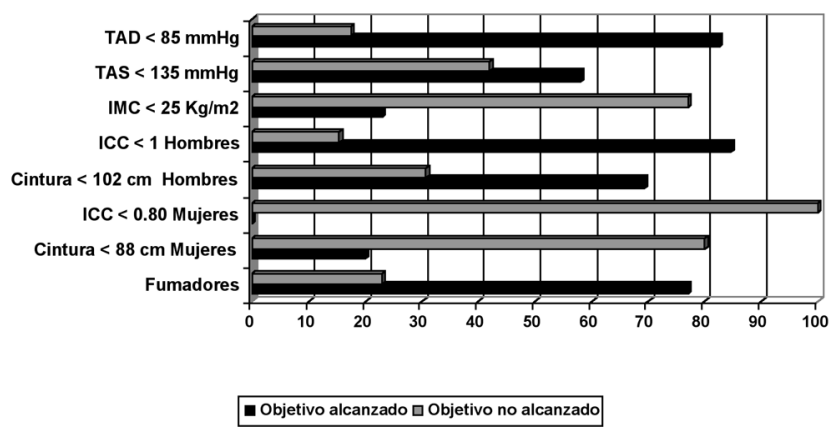

Fig. 2. Porcentaje de pacientes estudiados que alcanzan el objetivo de control óptimo. 
sos (5,7-12) para lograr prevenir, o al menos controlar, el desarrollo de estas complicaciones a partir de un buen control metabólico.

Con el fin de objetivar el grado de control de la DM2 y de los factores de riesgo asociados, se realizó este estudio en una población de diabéticos tipo 2 en Atención Primaria.

Del análisis de los resultados se deduce que este grupo de pacientes tiene, en general, un buen control metabólico, medido por la $\mathrm{HbA1c}(72,2 \%)$ y por la glucemia posprandial $(74,5 \%)$, no sucediendo lo mismo cuando valoramos la glucemia en ayunas $(33,3 \%)$, preferentemente en los varones (25\%). Esto quizás se deba al temor, por parte de los pacientes, a presentar hipoglucemias nocturnas, como han apuntado otros autores (13-15), que les limita el ajuste de la medicación antes de la cena, preferentemente en los tratados con insulina.

Observamos como los porcentajes de los FRV en ellos son muy elevados, concordantes con los de otras series (16). La mayoría presentan diferentes grados de obesidad. El $77 \%$ tienen un IMC $>25 \mathrm{~kg} / \mathrm{m}^{2}$ lo que constituye una alto riesgo para estos pacientes y una dificultad añadida para conseguir un mejor control metabólico. Le sigue en frecuencia la HTA con un $60 \%$. De ellos, sólo el $58 \%$ de mantienen un control óptimo si se trata de la PAS y sube al $82,6 \%$ si se trata de la PAD. Es probable que sea debido a que los médicos prestan mayor atención a la diastólica que a la sistólica por relacionarla más con el riesgo cardiovascular (16).

Aproximadamente la mitad presentan dislipemia típica de DM tipo 2 (40\%), sucediendo lo mismo que en la HTA, que pocos llegan a un control óptimo (26,8\% para la LDL-colesterol y un 58,9\% para el colesterol total) salvo para los triglicéridos donde la mayoría lo consigue $(93,2 \%)$. Sólo el $18 \%$ de los pacientes diagnosticados están sometidos a tratamiento hipolipemiante a pesar de que se ha demostrado claramente el efecto beneficioso de su reducción sobre el riesgo cardiovascular $(17,18)$

Así mismo es de señalar que siendo alto el porcentaje de pacientes con microalbuminuria (53\%), sea tan bajo el número de pacientes tratados con ARAII $(23,5 \%)$, Si tenemos en cuenta, además, el elevado porcentaje de hipertensos $(60 \%)$. La indicación de este tipo de medicamento, en estas situaciones, es prácticamente imprescindible, dado que se ha demostrado, de manera fehaciente, un efecto beneficioso tanto para la HTA como para la microalbuminuria, ya que dismunuyen el riesgo cardiovascular (19-25). La intervención sobre el eje renina-angiotensina es fundamental en el tratamiento del paciente de riesgo elevado con DM e HTA (26).

A pesar de que el abandono del hábito tabáquico supone una disminución del riesgo cardiovascular (27) en general y, en particular en estos pacientes, el $23 \%$ son fumadores, preferentemente los varones, cifra similar a las de otras series (16,28).

Aunque estos datos son de una población muy determinada, que no es posible extrapolar al resto de la población con DM2, si podemos deducir que todavía nos queda mucho camino por andar para lograr controlar mejor a los diabéticos tipo 2 y evitar la aparición, tan frecuente, de las complicaciones vasculares. La asociación de diferentes factores de riesgo a esta enfermedad hace que controlar una diabetes no sea sólo mirar el perfil glucémico, sino que tendremos que prestar además atención al resto de los factores, modificándoles, para obtener un control óptimo de cada uno de ellos. Creemos que la Atención Primaria juega un papel muy importante en el tratamiento de DM tipo 2, despistando precozmente los factores de riesgo y controlándolos lo más estrictamente posible. No es válido el diagnosticar si no sometemos al paciente a una terapéutica adecuada tanto de estilo de vida como medicamentosa.

\section{Bibliografía}

1. Banegas JR, Graciani A, Vilar F, Rodríguez-Artalejo F. Impacto de la diabetes en la mortalidad por enfermedades cardiovasculares en España. Med Clin Monogr (Barc) 2002; 3: 2-6

2. The World Health Report: life in the 21 st century, a vision for all. Ginebra: OMS 1988

3. Sociedad Española de Arterioesclerosis y de la Sociedad Española de Diabetes. Dislipemia diabética: Documento de Consenso de la Sociedad Española de Arterioesclerosis y de la Sociedad Española de Diabetes. Clin Invest Arterioesclerosis 1998; 10: 55-64

4. Labarthe DR. Epidemiology and prevention of cardiovascular disease. A global challenge. Gaitthersburg, Md, Aspen Publisher, 1998

5. UKPDS Group. Intensive blood glucose control with sulphonylurea or insulin compared with conventional tratment and risk complications in patients with type 2 diabetes (UKPDS 33). Lancet 1988; 352: 839-855.

6. O’Keefe J, Miles JM, Harris WH, Moe RM, McCallister BD. Improving the adverse cardiovascular prognosis of type 2 diabetes. Mayo Clin Proc 1999; 74: 171-180.

7. American Diabetes Association. Standars of medical care for patiens with diabetes mellitus. Diabetes Care 2002; 25 (Supl. 1): 533-549

8. European Diabeters Polcy Group. A strategy for arterial risk assessment and management in type 2 diabetes mellitus. European Arterial Risk Policy Group on behalf of the International Diabetes Federation European Region. Diabet Med 1997; 14: 611-621

9. Expert Panel on Detection Evaluation and Treatment of Haigh Blood Cholesteterol in Adults. Executive summary of the Third Report of the National Cholesterol Education Program (NCEP) Expert
Panel on Detection, evaluation anf Treatment of High Blood Cholesterol in Adults (Adults Treatment Panel III) JAMA 2001; 285: 2486-2497.

10. Seventh Report of the Joint National Committee on Prevention,Detection, Evaluation, and Treatment of High blood presure (VII JNC). Chobanian et al. Hypertension 2003; 42: 1206-1252.

11. National Institutes of Health. Clinical guideliness on the identification, evaluation and treatment of overweight and obesity in adults: the evidence report. Obes Res 1998; 6 (Supl. 2): 51-209.

12. Pablo Velasco PL, Picó Alfonso A. Guías de Actuación Clínica de la Diabetes Mellitus. Endocrinol Nutr 2003; 50 (Supl.): 1-71

13. American Diabetes Association: Standards of medical care for patients with diabetes mellitus. Diabetes Care 21 (Supl.1): S23-S31, 1998.

14. American Diabetes Association: Selfmonitoring of blood glucose (Consensus Statement). Diabetes Care 19 (Supl. 1): S62-S66, 1996.

15. Boland EA, Monsod T, Delucia M, Brand CA, Fernando S, Tamborlane WV: Limitations of conventional methods of self monitoring of blood glucose: lessons learned from 3 days of continuous glucose sensing in pediatric patients with type 1 diabetes. Diabetes Care 2001; 24: 1858-1862.

16. Cañizo Gómez FJ, Moreira-Andrés MN. Control de los factores de riesgo cardiovascular y diabetes mellitus tipo 2. ¿Seguimos las recomendaciones?. Educación Diabetológica Profesional 2003; XIII(2): 13-23

17. CARE Trial. Cardiovascular events and their reduction with pravastatin in diabetic and glucose intolerant myocardia infarction surviviors with average cholesterol levels. Circulation 1998; 98: 2513-9 
18. Scandinavian Simvastatin Survival Study Group: Randomized trial of cholesterol lowering in 4444 patients with coronary heart disease: the Scandinavian Simvastatin Survival Study (4S). Lancet 1995; 334: 1383 1389

19. Mogensen CI. Microalbuminuria as predictor of clinical diabetes nephropathy. Kidney Int 1987; 31: 673-80

20. Björn Dalhöf, Richard Devereux, Sverre E Kjseldm, Stevo Julius, Gareth Beevers,Ulf de Faire et al: Losartan Intervention For Endpoint reduction in hypertension. Comentarios al estudio: Intervención para reducción de eventos finales en hipertensión con Losartan. Estudio LIFE. Comentarios Martín Rioboo E, García Criado E, Fonseca del Pozo FJ. Medicina de Familia (And) 2002; 4: 56: 58

21. Parving HH, Lehnert H, Brochner-Motensen J, Gomis R, Andersen S, Arner P. Then effect of Ibersartanof the developmenton diabetic of nepfropathy in patiens with type 2 diabetes. N Engl J Med 2001; 345: 870-8

22. Mogensen CE,Neldam S, Tikkanen I, Oren S, ViskoperR, Watts RW et al: Randomised controlled trialof dual blockade of renin-angiotensin system in patients with hypertension, microalbuminuria, and non-insulin dependent diabetes: the candesartan and lisinopril microalbuminuria. (CALM) study. BMJ 2000; 321: 1440-4
23. Brenner Bm, Cooper Me, de Zeeuw D, Keane WF, Mitch WE, Parving $\mathrm{HH}$, et al. Effect of losartan on renal and cardiovascular outcomes in patients with type 2 diabetes and nepropathy. N Engl J Med 2001; 345: 253-9.

24. Lewis Ej, Hunsicker LG, Clsrke WR, Berl T, Pohl MA, Lewis JB, et al. Renoprotective effect of the angiotensin-receptor antagonist ibersartan in patients with nepropathy due to type 2 diabetes. N Engl J Med 2001; 345: 851-60

25. Aranda Lara P, Aranda Lara FJ, Aranda Granados PJ, López de Novales López E. Papel del bloqueo farmacológico del sistema renina-angiotensina en la protección del paciente con diabetes tipo 2. Hipertensión 2004; 20 (Supl. 2): 27-34

26. Gómez-Fernández P. Intervención sobre el sistema renina-angiotensina para la protección vascular del paciente hipertenso y en la nefropatía diabética. Rev Clin Esp 2004; 204: 596-600

27. Will J, Galuska D, Ford E, Mokdad A, Calle: Cigarette smoking and diabetes mellitus: evidence of a positive association from a large prospective study. Int J Epidemiol 2001; 30: 540-546

28. George PB, Tobin KJ, Corpues RA, Devlin WH, O Newill WW. Treatment of cardiac risk factors in diabetic patients: how well do we follow the Guidelines? Am Heart J 2001; 142: 857-863. 